\title{
AMCP Partnership Forum: Optimizing Prior Authorization for Appropriate Medication Selection
}

\begin{abstract}
SUMMARY
Prior authorization (PA) and step therapy (ST) are utilization management tools that have been in use by managed care organizations for decades. These processes require that health care providers obtain advanced approval to qualify a specific product for coverage from a health plan before it is delivered to the patient. These tools are intended to ensure that patients have access to evidence-based medications while payers remain good stewards of limited health care resources. PA and ST are growing in use to support appropriate use of medications and manage associated costs but may pose challenges related to administrative burden and access to care.

In June 2019, the Academy of Managed Care Pharmacy (AMCP) conducted a multistakeholder forum to identify processes for optimizing PA and ST utilization management programs. Health care leaders representing academia, health plans, integrated delivery systems, pharmacy benefit managers, employers, federal government agencies, national health care provider organizations, and patient advocacy organizations participated in the forum. Participants explored current operations of these programs, evaluated stakeholder perspectives on opportunities to improve these programs, and provided recommendations for next steps. They also reviewed current federal and state legislative and regulatory activities to reform PA and ST processes and offered guidance to support program improvements.

The goal of the forum was to gather stakeholder input to inform the development of recommendations to improve efficiencies around PA and ST processes; provide recommendations to address administrative burdens; increase the visibility of the clinical and economic value of PA and ST utilization management programs; collect, review, and disseminate data-driven, real-world experiences of PA programs that support clinical and economic value; collect and disseminate best practices around PA appeals and denial processes; and improve channels of communications between health insurance providers, health care professionals, and patients to minimize care delays and improve clarity of coverage authorization requirements.
\end{abstract}

J Manag Care Spec Pharm. 2020;26(1):55-62

Copyright $\odot 2020$, Academy of Managed Care Pharmacy. All rights reserved.

$\mathrm{P}$ rior authorization (PA) and step therapy (ST) are utilization management tools that are often applied to certain pharmacy and medical benefit services. Although such tools can support safe, appropriate, and cost-effective care, stakeholders have called for review of PA and ST programs to ensure that policies facilitate optimal patient outcomes while reducing costs and ensuring safety without creating unnecessary barriers to care. To address these issues, the Academy of Managed Care Pharmacy (AMCP) convened a forum in Alexandria, Virginia, on June 25-26, 2019.

As a foundation for the forum, a PA and utilization management concepts paper written by the AMCP Professional Practice Committee and PA and utilization management reform principles that were developed by a workgroup convened by the American Medical Association (AMA) were shared, and a survey of current practices from a convenience sample of health plans was fielded. ${ }^{1,2}$ Pre-forum readings informed the panel and roundtable discussions, which involved all forum participants. Real-world case studies were also discussed to illustrate nuances associated with best practices and challenges.

\section{Utilization Management and Clinical Decision Support:}

\section{Benefits and Limitations}

The need for decision support tools has only grown as the pace of innovation has accelerated and new clinical information emerges daily, and advances in care that improve outcomes may be associated with increased costs. ${ }^{3-6} \mathrm{PA}$ and ST are often used to support evidence-based coverage decisions, patient safety, and cost-effective medication use. ${ }^{1,7-9}$ However, these programs may create burdens for health care providers and patients..$^{10,11}$

Participants identified general characteristics of medications that could warrant the use of PA or ST and discussed various aspects of these programs, including descriptions, purpose, transparency, and appropriate use (Table 1). They also observed that, in some cases, the use of certain medications requires ancillary services (e.g., a genetic test) and that PA processes should include all required elements of the treatment.

\section{Administrative Burdens of PA}

The time and costs that prescribers, pharmacists, and patients dedicate to complying with PA programs have been cited as important challenges by professional societies, patient advocacy groups, and employers. ${ }^{11-17}$ Prescribers seek solutions that reduce the time required to determine the PA criteria for a specific plan, gather and submit information to the health plan, and follow up, particularly if an appeal is required.

Patients (and caregivers) need more efficient processes to support access to cost-effective, evidence-based therapy. There is a need for increased transparency in the PA process and timely communication so that patients can be better informed about the requirements and options for appeal.

\section{Calls to Improve PA}

Several organizations have released guiding principles for PA reform (Table 2). ${ }^{11-18}$ Key themes that have emerged across these organizations focus on the need for standardization in PA processes, evidence-based clinical criteria for protocol development, transparency of protocols, continuity of care, more effective communications to providers and patients, opportunities to appeal, and timeliness of processes. According to the pre-forum survey results, many plans report that they 


\section{TABLE 1 Participant Perspectives on Aspects of Utilization Management Programs}

\begin{tabular}{l|l}
\hline Aspect & \multicolumn{1}{c}{ Perspectives from Participants } \\
\hline $\begin{array}{l}\text { General characteristics of } \\
\text { medications that may lead to } \\
\text { utilization management }\end{array}$ & $\begin{array}{l}\text { - Specific safety concerns, including certain drug interactions } \\
\text { - Potential for off-label use } \\
\text { - Potential for misuse or abuse } \\
\text { - Limited distribution or special handling requirements } \\
\text { • Multiple indications across benefits (e.g., medical and cosmetic) }\end{array}$ \\
\hline Description of PA & $\begin{array}{l}\text { PA is a process for the provider to obtain approval for medications and/or services to qualify for coverage by the plan. It can } \\
\text { be a collaborative process that includes evidence-based criteria. }\end{array}$ \\
\hline Purpose of PA & $\begin{array}{l}\text { The purpose of PA is to ensure selection of safe, cost-effective, evidence-based treatment that maximizes therapeutic value. } \\
\text { Processes should be reasonable, accessible, and timely. }\end{array}$ \\
\hline $\begin{array}{l}\text { Disclosure and review of PA } \\
\text { requirements }\end{array}$ & $\begin{array}{l}\text { Communications need to be relevant, available in multiple formats, and easily accessible to patients, prescribers, and phar- } \\
\text { macists. There is a need to have some materials that are appropriate for patients, and health literacy and readability should } \\
\text { be considered when designing these communications. Patient, prescriber, and pharmacist communication should be incor- } \\
\text { porated throughout the PA process. }\end{array}$ \\
\hline $\begin{array}{l}\text { Appropriate use of PA and ST } \\
\text { protocols }\end{array}$ & $\begin{array}{l}\text { It is important for patients to receive access to cost-effective, evidence-based treatments. Incentives should be aligned in a } \\
\text { manner that influences total cost of care. Complying with appropriate PA and ST should not be challenging to operational- } \\
\text { ize for providers. }\end{array}$ \\
\hline
\end{tabular}

$P A=$ prior authorization; $S T=$ step therapy.

currently have practices that align with these principles, including that they collaborate with prescribers to develop criteria that are clinically sound and evidence based (unpublished data, Academy of Managed Care Pharmacy, 2019).

Participants reviewed publications, including those from AMCP and the workgroup convened by AMA, that explore how plans and providers can collaborate to reduce administrative burdens and costs associated with PA in its current form. ${ }^{1,16}$ Given that there is substantial alignment among organizations regarding factors for consideration, participants acknowledged that there are opportunities for collaboration to develop innovative solutions to improve PA processes and implement best practices.

Participants emphasized the need to focus on the patient's experience while addressing other stakeholder needs. They also recognized the need to return autonomy to the point-ofcare for prescribers who practice evidence-based medicine, while acknowledging that this requires accountability for costeffective care. Resources that remain abreast of the accelerative pace of new evidence generation will be an important tool to support evidence-based decision making.

Participants discussed examples of PA or utilization program elements that some states are considering and offered suggestions for fine-tuning these elements, including the description of PA, the purpose of PA and declarations, disclosure and review of PA requirements, and the appropriate use of PA and ST protocols (Table 1).

AMCP PA and Utilization Management Concepts in Managed Care Pharmacy. AMCP's Professional Practice Committee has developed 9 concepts for effective PA processes to support collaboration between prescribers and payers to ensure that patients receive timely access to cost-effective, evidence-based treatment (Table 3). ${ }^{1}$

During the forum, participants reviewed the detailed descriptions of each of these concepts and assessed alignment of these concepts with real-world examples of existing PA criteria. ${ }^{1}$ They identified several gaps between the concepts for effective PA processes and current practice. Opportunities for improvement emerged around addressing patient, prescriber, and pharmacist burden; transparency for stakeholders; clarity regarding requirements; variability among plan requirements; and reducing the complexity of criteria. Options for expanding the use of electronic PA (ePA) systems were also explored. However, automated decision support typically relies on binary data fields (e.g., yes/no). Organizations seeking to use ePA could structure their criteria to support binary data questions and responses.

\section{Evaluating Current Initiatives to Improve Processes}

Participants outlined criteria and identified barriers for recommended approaches to ensure that processes are patientcentered and reduce the administrative burden. The burden on prescribers, pharmacists, and patients is well described and complicated by a lack of consistency across formularies, requirements, and processes among plans, even for the same medication. ${ }^{13}$ The forum participants outlined factors that contribute to inconsistency in processes and formularies, including that employers have different priorities and offer different plans to their employees, regulations vary across states and plans by line of business, and organizational priorities and business operations of plans vary. 
TABLE 2 Key Themes in Guiding Principles for Utilization Management Reform 12-18

\begin{tabular}{|c|c|c|c|c|c|c|}
\hline \multirow[b]{2}{*}{ Theme } & \multicolumn{5}{|c|}{ Organization } & \multirow[b]{2}{*}{ AMCP } \\
\hline & $\begin{array}{c}\text { AMA Consensus } \\
\text { Statement }^{\mathrm{a}}\end{array}$ & ASCO & NPF & SAIM & NAF & \\
\hline Clinical criteria for protocol development & $\checkmark$ & $\checkmark$ & $\checkmark$ & $\checkmark$ & $\checkmark$ & $\checkmark$ \\
\hline Transparency of protocol & $\checkmark$ & $\checkmark$ & $\checkmark$ & $\checkmark$ & $\checkmark$ & $\checkmark$ \\
\hline Continuity of care & $\checkmark$ & $\checkmark$ & $\checkmark$ & $\checkmark$ & $\checkmark$ & $\checkmark$ \\
\hline Opportunity to appeal & $\checkmark$ & $\checkmark$ & $\checkmark$ & $\checkmark$ & $\checkmark$ & $\checkmark$ \\
\hline Flexibility for provider input & $\checkmark$ & $\checkmark$ & 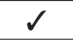 & $\checkmark$ & $\checkmark$ & $\checkmark$ \\
\hline Financial considerations & $\checkmark$ & - & - & - & - & $\checkmark$ \\
\hline Evaluation of program impact & $\checkmark$ & $\checkmark$ & - & $\checkmark$ & - & - \\
\hline Protocol updates & - & $\checkmark$ & - & $\checkmark$ & - & $\checkmark$ \\
\hline
\end{tabular}

Note: $\boldsymbol{V}$ =stakeholder discusses theme; -=stakeholder silent on theme.

aSigning organizations included American Hospital Association, America's Health Insurance Plans, American Medical Association, American Pharmacists Association, BlueCross BlueShield Association, and Medical Group Management Association.

$A M A=$ American Medical Association, ASCO=American Society of Clinical Oncologists, NPF= National Psoriasis Foundation, SAIM = State Access to Innovative Medicines Coalition, NAF = National Arthritis Foundation.

Participants provided insights on how to improve existing recommendations and guidance for the use of potential solutions, including pharmacist-initiated PA, sunset programs, and gold card programs. Several proposed strategies could reduce administrative burdens.

\section{Sequencing Therapy: ST and Clinical Pathways}

Participants discussed the similarities of characteristics across ST and clinical pathway programs. They identified challenges associated with sequencing programs, including lack of consistent descriptions across health plans and gaps in communication between health plans, patients, and providers. Processes for exempting patients from ST and the process to initiate an appeal were also cited as challenges.

To improve sequencing programs, participants suggested the development of criteria that would allow prescribers to (a) answer "yes" or "no" to a series of questions and (b) rely more on provider attestations to avoid requirements for submitting detailed patient records (e.g., PDFs), which may not be interoperable with current technology systems. Clinical practice guidelines could be an effective tool for the development of criteria if they are available, current, and sufficiently specific for the disease being treated and could support consistency among plans.

Participants observed that value-based arrangements that place providers at risk for total cost of care support incentives to provide cost-effective care. Accountability for the financial and clinical outcomes of care, combined with robust quality improvement programs, may support increased autonomy for prescribers and could result in a reduction in PA or ST requirements. As the health system continues the trend toward value-based purchasing, PA may increasingly shift to acting as a decision support tool.
Participants also addressed challenges related to the durability of approvals. Ideally, patients are only required to progress through ST requirements once. However, when patients switch employers or insurance plans or have a lapse in therapy, they may be required to document that they have been through ST processes. Because of the effect on patient outcomes and safety, some health plans "grandfather" patients, allowing those who are already responding well to a therapy to continue treatment without interruptions. However, challenges transferring patient data among health plans may affect the ability of payers to provide timely "grandfathering" of existing treatments.

The general perspective of forum participants was that patients established on therapy should have continuity of care and not be required to step through new or additional therapies or criteria to remain on treatment that is effective for them. However, communication across payers to facilitate continuity of care is a recognized challenge. As greater sharing of data occurs in the health care system, stakeholders will need to support increased sharing of patient history information, including medication history, to assist in the review of ST requirements.

\section{PA Denial and Appeal Processes}

Stakeholders seek transparency and improved communication throughout PA denial and appeal processes. Effective communication among health plans, health care providers, and patients is necessary to ensure timely completion of PA requests and to minimize care delays.

Participants discussed opportunities to improve the denial and appeal process, including timelines for these processes, the qualifications of the individual who reviews requests, and the need for clear patient-centric communication about reasons for denial. Regulatory requirements exist that define timelines for 


TABLE 3 $\begin{aligned} & \text { AMCP Prior Authorization and } \\ & \text { Utilization Management Concepts in } \\ & \text { Managed Care Pharmacy }\end{aligned}$
1. Patient safety and appropriate medication use
2. Clinical decision making
3. Evidence-based review criteria
4. Automated decision support
5. Transparency and advance notice
6. Emergency access
7. Provider collaboration
8. Need for timeliness and avoiding disruptions in therapy
9. Cost-effectiveness and value
AMCP=Academy of Managed Care Pharmacy.

approvals and denials of requests, exceptions, and appeals. For example, for an initial coverage determination, Medicare Part D requires a response from the reviewer within 72 hours, or 24 hours for expedited reviews. ${ }^{19}$ Participants identified an opportunity to improve communication consistency and approval timelines across health plans and lines of business to facilitate timely resolution of PA requests and minimize care delays.

To inform, refine, and improve utilization management programs, several stakeholders have called for health plans to improve transparency by listing metrics on their websites such as PA volume, approval rates, and denial rates. Participants observed that details regarding the reasons for PA denials (e.g., medical necessity not demonstrated, incomplete PA submission, and safety issues) would be needed to provide important context for interpreting statistical data.

\section{Pharmacist-Initiated PA}

Participants reviewed the operations of a pharmacist-initiated PA clinic and discussed various aspects of the process (Figure 1). A key benefit is that pharmacists have access to information from the patient's prescription history to supply to the plan to process the PA. Ideally, pharmacists will also have direct access to the patient's electronic health record (EHR) to obtain additional details that may be required, such as diagnosis or laboratory values. Finally, the greater accessibility of pharmacists to patients, particularly on evenings and weekends, was cited as a benefit for patients, who may have questions throughout the process.

A similar solution currently in practice is the use of centralized PA teams. This approach allows the administrative burden to be shifted away from physicians to other health care professionals, such as pharmacists, nurses, and pharmacy technicians, who specialize in performing PA support services. Compared with usual care, pharmacy-led interventions in PA processing significantly improve time to PA approval, time to first fill, and time to pick up. ${ }^{20}$ That further examination of pharmacist responsibilities, as well as the potential for improved workflow efficiencies, is necessary for advancing pharmacist-initiated PAs.

\section{Evaluating PA Criteria}

Regular review of the metrics associated with approvals and denials for products and services that are subject to PA requirements can help identify therapies that no longer warrant PA. For example, low variation in use or low PA denial rates may suggest that the criteria are no longer clinically relevant. Ideally, health plans will track metrics about rationales for denials and reasons for overturning denials on appeal to make informed decisions about criteria effectiveness. Regular review can help identify services, particularly new and emerging therapies, where PA may be warranted.

Some programs, including sunset and gold card programs, remove PA requirements either entirely or for a subset of prescribers. Although sunset and gold card programs are an option that health plans can implement to reduce provider burden, participants cautioned against the universal removal of PA criteria due to the possibility that this could have an undesirable effect on prescribing behavior. ${ }^{21}$ In addition, participants observed that the complexity of the health care system may require the use of PA programs in certain circumstances, such as when there is a reinsurance program (a form of insurance that insurance companies purchase to manage risks of covering patients with high medical costs) or a safety concern.

Sunset Programs. Participants discussed key considerations for establishing PA sunset programs (Figure 2). ${ }^{22}$ High PA approval rates alone were generally not considered sufficient to justify a sunset program, as they may indicate that prescribers are familiar with the requirements of the program. Participants noted that safety is an important reason to continue a PA regardless of approval rates but that the existence of a Risk Evaluation and Mitigation Strategy program for certain prescribing requirements could support removal of redundant PA criteria. They commented that the frequency of appeals should also be considered when developing a sunset program.

Gold Card Programs. Gold card programs differentiate the application of PA and/or ST based on provider performance on specific measures (e.g., adherence to formulary, evidence-based medical practices), or participation in a value-based agreement with the health insurance provider. ${ }^{23,24}$ Such programs minimize administrative burdens for prescribers who meet the program's requirements but may also have an undesirable effect on prescribing behavior. ${ }^{21}$

\section{Technology and Real-World HIT Solutions}

Participants discussed real-world applications and benefits of electronic PA (ePA) processes compared with fax and telephonic processes. An important advantage of ePA is that it can automate many steps of the process. ePA is more commonly 
FIGURE 1 Description of Pharmacist-Initiated PA in a Centralized Refill Clinic ${ }^{20}$

When a new medication is prescribed for a patient, the clinic pharmacist would:

- Check the patient's insurance plan to determine if a PA is required.

- The pharmacist, working under a collaborative practice agreement, would either perform a therapeutic interchange or initiate a PA request. When a PA is needed, the pharmacist accesses the patient's medical record to gather required information.

- Appropriate forms are completed (either electronically or manually) and a letter of medical necessity, written by the pharmacist, accompanies each form. The letter of medical necessity covers patient-specific reasons why the prescribed medication is preferred over alternative formulary options.

- After the determination by the insurance company is made, this information is communicated to the pharmacist who informs the prescriber and patient.

- In the event of a denial, the pharmacist recommends alternative options to the prescriber and/or patient, including another medication, patient-assistance programs, coupons, or vouchers.

$P A=$ prior authorization.

used for drugs covered under the pharmacy benefit compared with those covered under the medical benefit (e.g., certain drugs that are injected or infused in medical settings). Within the medical benefit, ePA is used to address procedures services and devices; it could be more widely used for drugs covered under the medical benefit, including specialty drugs. ${ }^{25}$ However, interoperability challenges across different technology systems remain.

Participants considered how ePA could help reduce burdens on patients and providers. Ideally, ePA would automatically connect with the EHR in real time to populate required information so that it does not have to be entered manually by prescribers. Participants also recommended that ideally the e-prescribing systems would be able to perform a real-time benefit check at the point of prescribing, similar to electronic claim adjudication performed in the pharmacy.

The ability of ePA to reduce the burdens on providers and patients was explored. Participants observed that, if PA is required, ePA facilitates a more seamless process for prescribers and patients. Further, they noted that it can reduce the time for approval from days or weeks to a few minutes and reduces the rate of prescriptions that are abandoned by patients. Participants observed that ePA programs that function as decision-support tools are available, but not all providers have access to these programs and not all health plans have the required technology.

Participants supported consistency in the structure of web access to ePA to minimize administrative burdens and discussed the possibility of standardization of user interfaces and processes to improve consistency among programs. The development of industrywide standards was suggested as an approach to implement this solution. Participants recommended collaborating with technology vendors to support standardization and develop incentives to improve use of tools that currently exist.

\section{Legislative Activities and Reform Principles}

Several states have legislation that includes patient protections when PA or ST is implemented. Most state-level legislation has provisions for clear exceptions and appeals processes for PA and ST. Common areas of focus include timelines and appeals processes, but there is variability in how these concepts are addressed. For example, state-required time limits for the review of appeals range from 24 to 72 hours. A few laws include specific provisions for patients with advanced metastatic cancer; others are not specific to particular conditions.

Legislation currently being considered by states generally reflects guidance from stakeholder principles and statements. Examples of issues that are being addressed in various legislative initiatives include the following:

- Clinical criteria must be evidence based, current, consider provider input, and accommodate unique patient populations.

- ST protocols should be accessible, include electronic processes for communication, and have flexibility to accommodate individual needs. Patients should not be required to repeat treatment steps.

- Interruptions in care should be avoided.

- Appeals processes should be clear and readily accessible.

- Prescribers have the option to request overrides with appropriate justification.

Federal legislation could address some of the existing loopholes at the state level and provide some consistency for health plans. At the federal level, The Safe Step Act (H.R. 2279), currently under consideration, amends the Employee Retirement Income Security Act of 1974 to require an exceptions process for ST programs. The proposed legislation calls for clear processes for exceptions, lists requirements for granting exceptions, and requires that exceptions be granted within 72 hours (24 hours in urgent situations). However, participants observed that deadlines can result in denials if plans are unable to obtain the information needed to process the PA within the required timeline. 


\section{FIGURE 2 Description of PA Sunset Programs 22}

A PA sunset program includes the regular review of the list of medical services and prescription drugs that are subject to PA requirements to help identify therapies that no longer warrant PA due to, for example, low variation in utilization or low PA denial rates. Regular review can also help identify services, particularly new and emerging therapies, where PA may be warranted due to a lack of evidence on effectiveness or safety concerns.

Plans considering implementing a PA sunset program should consider using multiple evaluation criteria and data points and ensure that the metrics are powered appropriately to assess utilization, especially for medications used in small populations or for rare diseases.

\section{Criteria for review could include:}

- Review of medical services and prescription drugs requiring PA on at least a consistent input of ____ [list key internal and external stakeholders]

- Revision of PA requirements should be based on data and up-to-date clinical criteria, including:

- PA denial rates less than

- PA approval rates more than

- PA appeal rates

- PA request rates less than [\%] $[\%]$

- Financial impact [savings over time period]

- Treatment options in therapeutic class

- Safety [boxed warnings, REMS] [FDA approvals, new generics]

- Updated clinical information, such as [guidelines] [NCQA, state regulations]

- Plans should communicate changes to the lists of medical services and prescription drugs requiring PA via (a) provider-accessible [platform, websites] ; (b) at least____ [time frequency] ; and (c) [other]

FDA =U.S. Food and Drug Administration; NCQA = National Committee for Quality Assurance; PA = prior authorization; REMS=Risk Evaluation and Mitigation Strategy.

Participants stressed that legislative solutions can be difficult to achieve and alternative approaches may be needed. Reporting requirements for certain plans (e.g., Medicare Part D or Medicare Advantage plans) and collaboration with accrediting bodies were mentioned as examples of alternative strategies that could be used to drive change.

Some participants proposed that AMCP lead a discussion around the creation of model legislative language that considers all stakeholders. They stressed that legislation be written in a manner that is broad enough to be implementable and operational as practice evolves, and participants recommended that accrediting bodies and industry groups be responsible for addressing more detailed aspects of the processes.

\section{Conclusions}

Work to improve the current PA and ST processes requires ongoing efforts and collaboration among payers, prescribers, pharmacists, and patient groups to ensure that solutions meet the needs of all stakeholders. Organizations looking to improve PA and ST programs will need to consider how to automate processes, increase transparency, and remove the need for PA where appropriate while also promoting evidencebased prescribing. The importance of maintaining prescriber autonomy coupled with accountability, so that evidence-based and cost-effective treatments are prescribed, was highlighted throughout the forum.

Expanding the use of health information technology tools, such as ePA, is a best practice to reduce the administrative burdens of PA programs. Broader implementation and utilization of health information technology solutions by health care entities is needed to enable a more efficient health care system.

Finally, legislative and regulatory approaches and collaborative initiatives that address challenges associated with PA and ST will need to be mindful of the needs of all stakeholders while focusing on ensuring that patient care is optimized in an evidence-based, cost-effective manner.

\section{Forum Participants}

AMANDA BAIN, PharmD, MPH, MBA, Director, Pharmacy and Care Management, The Ohio State University Health Plan; HEATHER BONOME, PharmD, Director of Pharmacy, URAC; MICHAEL BRODEUR, RPh, MBA, Director, Clinical Drug Assessment, Aetna Pharmacy Management; ELIZABETH BRUSIG, PharmD, MBA, Clinical Pharmacy Specialist, Optima Health Plan; ANNE BURNS, BPharm, RPh, Vice President, Professional Affairs, American Pharmacists Association; SUSAN DOWNARD, RPh, Director, Clinical Pharmacy and Drug Utilization Management, Tufts Health Plan; MELISSA FALCONE, MBA, Vice President, Patient Services and Reimbursement, Mallinckrodt Pharmaceuticals; JENNIFER GRAFF, PharmD, Vice President, Comparative Effectiveness Research, National Pharmaceutical Council; ERIC GRATIAS, MD, FAAP, Senior Vice President and Chief Medical Officer, Oncology/Laboratory/Specialty Drug Services, eviCore healthcare; JULIE HESSICK, RPh, Senior Director, Business Development, OneOme; SHARONA HOFFMAN, JD, LLM, SJD, Professor of Law and Bioethics, Case Western Reserve University School of Law; JIM HOPSICKER, RPh, MBA, Senior Leader, 
Pharmacy Strategy and Management, MVP Health Care; ANNA HYDE, Vice President of Advocacy and Access, Arthritis Foundation; PAUL JEFFREY, PharmD, Director of Pharmacy, MassHealth/UMass Medical School, Office of Clinical Affairs; TARUJA KARMARKAR, MHS, PhD, Postdoctoral Fellow in Health Policy, National Pharmaceutical Council/Duke-Margolis Center for Health Policy; JOCELYN KEEGAN, Payer Practice Lead, Point of Care Partners; STACY KNOX, PharmD, BCPS, BCACP, Pharmacist Supervisor, University of California Davis Health; LISA LE GETTE, RPh, MBA, Director, Government Affairs, Express Scripts; AMY LUGO, PharmD, BCPS, BC-ADM, FAPhA, Clinical Specialist and Formulary Manager, Defense Health Agency/DoD/TRICARE; CRAIG MATTSON, MS, MBA, RPh, Retired, Prime Therapeutics; HEATHER MCCOMAS, PharmD, Director, American Medical Association; DANA MCCORMICK, RPh, Director Pharmacy, Blue Cross Blue Shield of Texas; GARY OWENS, MD, President, Gary Owens Associates; STEVEN PESKIN, MD, Executive Medical Director, Horizon Blue Cross Blue Shield of New Jersey; KIRSTEN POWELL, Senior Director, Head Public Policy and Reimbursement, Takeda Pharmaceuticals America; MINDY PRASAD, PharmD, Manager, Pharmacy Benefit Utilization Management and Strategy, Blue Cross Blue Shield of Michigan; ELIZABETH SAMPSEL, PharmD, MBA, BCPS, Vice President, Payer Strategy and Relations, Dymaxium; ANNIE SCHUSTER, PharmD, Senior Director, Clinical Program, Cigna; CRAIG STERN, RPh, PharmD, MBA, FASCP, FASHP, FICA, FLMI, FAMCP, FCPhA, President, Pro Pharma Pharmaceutical Consultants; PATRICK STONE, Vice President, Government Relations and Advocacy, National Psoriasis Foundation; DANIEL TJIOE, PharmD, Medical Affairs Director, Merck; MICHAEL TOCCO, RPh, MEd, President, Integrated Pharmacy Solutions; LAURA TOPOR, President, Granada Health; KRYSTALYN WEAVER, PharmD, Vice President, Policy, National Alliance of State Pharmacy Associations; NICK WEBBER, Principal Business Advisor, Product Innovation, Surescripts; and SUSAN WINCKLER, RPh, Esq, President, Leavitt Partners Solutions, Leavitt Partners.

\section{AMCP Staff Participants}

CHRISTINE COOPER, PharmD, Associate Director of Educational Programs; JUDY CRESPI-LOFTON, MS, Writer, JCL Communications; JULIAN GREER, CMP, Senior Manager, Meetings and Forums; NEAL LEARNER, Media Relations and Editorial Director; NOREEN MATTHEWS, BSN, MBA, Senior Consultant, Strategic Alliances and Corporate Services; ZAIN MADHANI, Program Manager, Policy and Government Relations; CYNTHIA REILLY, MS, BSPharm, Chief Operating Officer; TERRY RICHARDSON, PharmD, BCACP, Senior Pharmacist Consultant; PHILIP SCHNEIDER, BS, MA, MS, Consultant to Executive Director of AMCP Foundation; and TRICIA LEE WILKINS ROLLE, PharmD, MS, PhD, Director, Pharmacy Affairs.

CORRESPONDENCE: Cynthia Reilly, MS, BSPharm, Chief Operating Officer, Academy of Managed Care Pharmacy, 675 N. Washington, St., Ste. 220, Alexandria, VA 22314. Tel.: 703.684.2600; E-mail: creilly@amcp.org.

\section{DISCLOSURES}

This AMCP Partnership Forum was sponsored by Mallinckrodt Pharmaceuticals, Merck, the National Pharmaceutical Council, and Takeda. These proceedings were prepared as a summary of what occurred at the forum to represent common themes; they are not necessarily endorsed by all attendees nor should they be construed as reflecting group consensus.

\section{ACKNOWLEDGMENTS}

These proceedings were written by Judy Crespi-Lofton, Medical Writer and Consultant, JCL Communications, who declares no conflict of interest, real or apparent, with any product or service mentioned in this article.

\section{REFERENCES}

1. 2018-2019 Academy of Managed Care Pharmacy Professional Practice Committee. Prior authorization and utilization management concepts in managed care pharmacy. J Manag Care Spec Pharm. 2019;25(6):641-44. Available at: https://www.jmcp.org/doi/10.18553/jmcp.2019.19069.

2. American Medical Association, American Academy of Child and Adolescent Psychiatry, American Academy of Dermatology, et al. Prior authorization and utilization management reform principles. Available at: https://www.ama-assn.org/system/files/2019-06/principles-with-signatorypage-for-slsc.pdf. Accessed December 3, 2019.

3. Ramsey SD, Fedorenko C, Chauhan R, et al. Baseline estimates of adherence to American Society of Clinical Oncology/American Board of Internal Medicine choosing wisely initiative among patients with cancer enrolled with a large regional commercial health insurer. J Oncol Pract. 2015;11(4):338-43.

4. Rocque G, Blayney DW, Jahanzeb M, et al. Choosing wisely in oncology: are we ready for value-based care? J Oncol Pract. 2017;13(11):e935-e943.

5. Roman BR, Patel SG, Wang MB, et al. Guideline familiarity predicts variation in self-reported use of routine surveillance PET/CT by physicians who treat head and neck cancer. J Natl Compr Canc Netw. 2015;13(1):69-77.

6. Densen P. Challenges and opportunities facing medical education. Trans Am Clin Climatol Assoc. 2011;122:48-58.

7. Mishuk AU, Fasina I, Qian J. Impact of U.S. federal and state generic drug policies on drug use, spending, and patient outcomes-a systematic review. Res Social Adm Pharm. August 17, 2019 [Epub ahead of print].

8. Keast SL, Holderread B, Cothran T, Skrepnek GH. Assessment of the effect of an enhanced prior authorization and management program in a United States Medicaid program on chronic hepatitis C treatment adherence and cost. J Am Pharm Assoc (2003). 2018;58(5):485-91.

9. Butcher L. Seeing it from both sides now. Manag Care. 2017;26(11):18-24.

10. Kirkwood MK, Hanley A, Bruinooge SS, et al. The state of oncology practice in America, 2018: results of the ASCO Practice Census Survey. J Oncol Pract. 2018;14(7):e412-e420.

11. Hoffman S. Step therapy: legal and ethical implications of a cost-cutting measure. Food \& Drug L. J. 2018;73:38-65 . Available at: https://scholarlycommons.law.case.edu/faculty_publications/2009. Accessed December 3, 2019.

12. American Society of Clinical Oncology. American Society of Clinical Oncology statement on the impact of utilization management policies for cancer drug therapies. J Oncol Pract. 2017;13(11):758-62.

13. American Medical Association. 2018 AMA Prior Authorization (PA) Physician Survey. Available at: https://www.ama-assn.org/system/files/201902/prior-auth-2018.pdf. Accessed December 3, 2019.

14. National Psoriasis Foundation. Position statement on access to care. October 2018. Available at: https://www.psoriasis.org/sites/default/files/ position_statement_on_access_to_care_revisions_final_10_03_18.pdf. Accessed December 3, 2019. 
15. Arthritis Foundation. Prior authorization. Available at: https://www. arthritis.org/advocate/our-legislative-position-statements/prior-authorization. php. Accessed December 3, 2019.

16. American Hospital Association, America's Health Insurance Plans, American Medical Association, American Pharmacists Association, BlueCross BlueShield Association, Medical Group Management Association. Consensus statement on improving the prior authorization process. Available at: https://www.ama-assn.org/sites/ama-assn.org/files/corp/mediabrowser/public/arc-public/prior-authorization-consensus-statement.pdf. Accessed December 3, 2019.

17. State Access to Innovative Medicines Coalition. Step therapy. Available at: https://www.saimcoalition.org/state-access-to-innovative-medicines/steptherapy/. Accessed December 3, 2019.

18. Karmarkar T. Legislative activities and reform principles in prior authorization: where are we today? Presented at: AMCP Partnership Forum: Optimizing prior authorization for appropriate medication selection; June 26, 2019; Alexandria, VA.

19. Centers for Medicare \& Medicaid Services. Medicare prescription drug (Part D) coverage determination/appeals process [chart]. Available at: https://www.cms.gov/Medicare/Appeals-and-Grievances/ MedPrescriptDrugApplGriev/Downloads/Flowchart-Medicare-Part-D.pdf. Accessed December 3, 2019.
20. Cutler T, She Y, Barca J, et al. Impact of pharmacy intervention on prior authorization success and efficiency at a university medical center. J Manag Care Spec Pharm. 2016;22(10):1167-71. Available at: https://www.jmcp.org/ doi/10.18553/jmcp.2016.22.10.1167.

21. Minchin M, Roland M, Richardson J, et al. Quality of care in the United Kingdom after removal of financial incentives. N Engl J Med. 2018;379(10):948-57

22. Erickson SM, Rockwern B, Koltov M, et al. Putting patients first by reducing administrative tasks in health care: a position paper of the American College of Physicians. Ann Intern Med. 2017;166(9):659-61.

23. Washington State Office of the Insurance Commissioner. Optimizing prior authorization, also known as goldcarding. Stakeholder draft. September 14, 2017. Available at: https://www.insurance.wa.gov/sites/default/files/201709/2017-07-stakeholder-draft-sept-14.pdf. Accessed December 3, 2019.

24. Blue Cross Blue Shield of Michigan, Blue Care Network Radiation. Oncology Gold Carding Program. Available at: https://mroqc.sites.uofmhosting.net/sites/default/files/MROQC\%20Gold\%20Carding\%20FAQ\%20 v.3.28.18_1.pdf. Accessed December 3, 2019.

25. Kleinberg K, Keegan J, Schueth T, Solomone M. Prior authorization's real benefits: reducing frictional costs and speeding time to therapy. Point of Care Partners. May 2019. Available at: https://www.pocp.com/downloads/ ePAReport. Accessed December 3, 2019. 